\title{
Excluded Volume Causes Integer and Fractional Plateaus in Colloidal Ratchet Currents
}

\author{
Pietro Tierno ${ }^{1,2, *}$ and Thomas M. Fischer ${ }^{3}$ \\ ${ }^{1}$ Estructura i Constituents de la Matèria, Universitat de Barcelona, Avenida Diagonal 647, 08028 Barcelona, Spain \\ ${ }^{2}$ Institut de Nanociència i Nanotecnologia IN2UB, Universitat de Barcelona, 08028 Barcelona, Spain \\ ${ }^{3}$ Institute of Physics, Universität Bayreuth, 95440 Bayreuth, Germany
}

(Received 28 November 2013; published 31 January 2014)

\begin{abstract}
We study the collective transport of paramagnetic colloids driven above a magnetic bubble lattice by an external rotating magnetic field. We measure a direct ratchet current which rises in integer and fractional steps with the field amplitude. The stepwise increase is caused by excluded volume interactions between the particles, which form composite clusters above the bubbles with mobile and immobile occupation sites. Transient energy minima located at the interstitials between the bubbles cause the colloids to hop from one composite cluster to the next with synchronous and period doubled modes of transport. The colloidal current may be polarized to make selective use of type up or type down interstitials.
\end{abstract}

The emergence of quantized steps in the current of a driven system is a fascinating phenomenon in condensed matter, occurring in a broad range of systems such as charge density waves [1,2], driven vortex lattices [3,4], sliding frictional surfaces [5], or electronic tunneling [6,7]. The impossibility of visualizing condensed matter quasiparticles has triggered the use of alternative model systems to unveil the basic mechanism leading to such transport behavior. Colloidal particles with accessible length scale and dynamics, represent a versatile model system with tuneable interactions [8-10]. In particular, hard sphere interactions creating excluded volume [11-13] are relevant for the rheological properties of colloidal dispersions $[14,15]$, and they dominate the dynamics near the colloidal glass transition [16,17]. Here we show that the excluded volume between paramagnetic colloids driven above a magnetic bubble lattice causes a series of discrete plateaus in the particle current, separated by steps where some particles abruptly lose or gain mobility. Integer plateaus result from particles moving synchronously with the driving field, while fractional plateaus arise from nonlinear period doubling with particles moving only every second cycle. In contrast to many ratchet mechanisms [18-20] which operate under negligible particle-particle interactions, we introduce a colloidal ratchet where quantized transport phenomena arise due to excluded volume between the particles.

We use monodisperse polystyrene paramagnetic colloids dispersed in deionized water and moving on top of ferrite garnet film (FGF) characterized by a triangular lattice of magnetic bubble domains [21] [Fig. 1(a)]. The FGF film exerts magnetic attraction to the paramagnetic colloids, and confines their motion in two dimensions. A direct ratchet current is obtained by modulating the heterogeneous stray field of the FGF with an external field elliptically polarized in the $(x, z)$ plane, $\boldsymbol{H}_{\mathrm{ext}} \equiv\left(H_{x} \sin (\omega t), 0, H_{z} \cos (\omega t)\right)$ (more details are given in [22]). Here $H_{z}$ indicates the component perpendicular to the FGF, $H_{x}$ the parallel, and $\omega$ the angular frequency. Figure 1(c) illustrates how the potential energy landscape of a paramagnetic colloid is altered by the applied field during one field cycle. The external field modulates the potential and increases or decreases the potential wells of the magnetic bubbles when it is parallel or antiparallel to the bubble magnetization. Before the field is oriented completely antiparallel to the bubble, two additional energy wells per unit cell nucleate in the marked blue and red interstitial regions in Fig. 1(b). Due to the parallel component of the field, $H_{x}$, the nucleation sites are displaced from the centre of the three surrounding bubbles and are located in the proximity of the particle "emitter" bubble. The preference for one of the three bubbles vanishes for completely antiparallel field orientations $H_{x}=0$, and reverses when the parallel field orients mirror symmetrically $H_{x, \text { annihil }}=-H_{x, \text { nucl }}$ with respect to the orientation during the nucleation. The interstitial wells hence nucleate near a bubble which becomes an emitter of particles and they annihilate near the collector bubble i.e., the neighboring bubble in the transport direction. The chirality of the external magnetic field modulation ensures unidirectional motion from the emitter bubble toward the collector bubble. A directed ratchet current is induced when a particle moves from an emitter bubble into a collector bubble via the interstitial region.

The collective effect of the excluded volume on the transport for small $(1 \mu \mathrm{m})$ particles is shown in Fig. 2(a) (movie, ad Fig. 2.WMV [22]). Individual particles will reside in the bubble wells, while bubbles filled with several particles generate a composite cluster with a radius, $r_{\text {cluster }}$. The green shaded column in Fig. 2(a) separates the unloaded lattice, on the right, from a series of loaded bubbles thereby forming composite clusters, on the left. The particles are transported from the left bubbles passing via the 


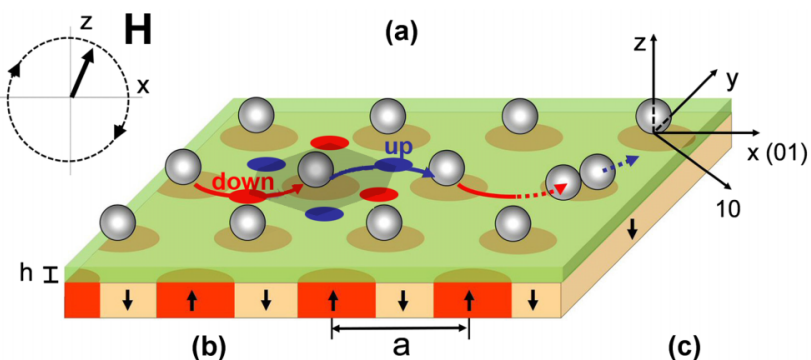

(b)
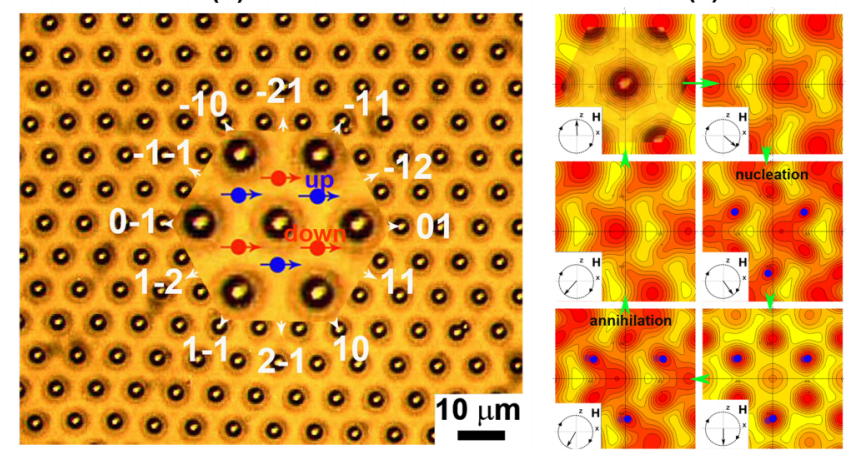

FIG. 1 (color online). (a) Schematic of the FGF film $(a=8.6 \mu \mathrm{m})$ covered with a polymer film $(h=1 \mu \mathrm{m})$, with one Wigner-Seitz unit cell shaded. Potential particle paths between the bubbles pass the type up (blue) and type down (red) interstitial regions. (b) Polarization microscopy image of the FGF loaded with one particle per unit cell. Crystal directions are indicated in white, type up and type down interstitial wells are marked in blue and in red, and nucleate at the beginning of the arrows, annihilate at the end. Central region has been magnified for clarity. (c) Magnetic potential energy of a paramagnetic particle during different phases of the applied field (inset). Energy maxima are colored in yellow, minima in red.

interstitial regions to the marked unfilled bubble, which they reach at $t=0.1 \mathrm{~s}$, and they start to grow a new composite cluster. For small values of $r_{\text {cluster }}$, this composite cluster is unable to emit particles into the interstitial wells to its right, and no net current is observed. For $t>1.35 \mathrm{~s}$, the cluster size surmounts a critical value, $r_{\text {cluster }}>r_{c} \sim 2 \mu \mathrm{m}$, and the particles located within $r_{c}$ remain immobile, while excess particles are emitted to the right into an interstitial and transported to the next collector bubble. In each cycle, the excess particles flow via the interstitials, filling more bubbles till reaching a stationary state that corresponds to a particle loading of $r_{\text {cluster }} \approx r_{c}$ for all bubbles.

We measure the current $\mathbf{j}(\rho)=\rho \mathbf{v}$ of particles with velocity $\mathbf{v}$ as a function of the particle number area density $\rho$. Figure 2(c) shows the dimensionless current, $\tilde{j}=$ $2 \pi A j / \omega a_{1}$ versus the dimensionless density $\tilde{\rho}=\rho A$, where $A=a_{1}^{2} \sin (\pi / 3)$ is the area of the unit cell, $a_{1}$ is the length of the unit vector in the 01 direction. We observe no current below an average loading of $\tilde{\rho} \leq 8$ particles per unit cell. Only if $\tilde{\rho}>N_{c}=8$, a net current is observed, which linearly increases with the density as $\tilde{j}=\tilde{\rho}-N_{c}$. Eight particles in each unit cell remain immobile while only the excess particles contribute to the current.
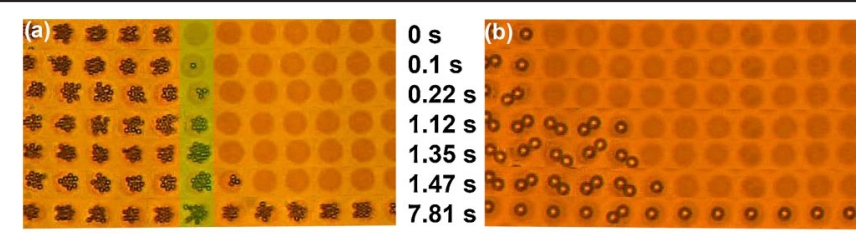

(c)

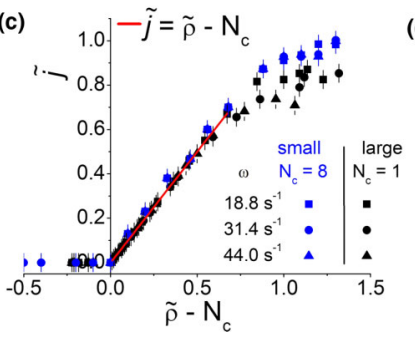

(d)

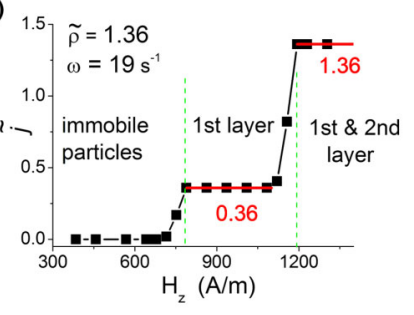

FIG. 2 (color online). (a) Time sequence of polarization microscopy images showing a row of bubbles subsequently filled with paramagnetic particles of diameter $d=1 \mu \mathrm{m}$ (a), and $d=$ $2.8 \mu \mathrm{m}$ (b). Particle motion occurs from left to right. In (a) the magnetic bubble in the shaded column requires a filling of $\tilde{\rho}>8$ particles to emit particles towards the next bubble. In (b) the particle transport occurs when the bubbles are filled with $\tilde{\rho}>1$ particles. (c) Normalized current $\tilde{j}$ for particles of size $d=1 \mu \mathrm{m}$, blue symbols, and of $d=2.8 \mu \mathrm{m}$, black symbols, as a function of the overloading, $\tilde{\rho}-N_{c}$ (d) Partially filled integer plateaus in the normalized current for $d=2.8 \mu \mathrm{m}$ particles as a function of the amplitude of $H_{z}$. (movie, ad Fig. 2.WMV [22]).

While small particles require the formation of highly populated clusters to produce net flow, we can simplify the complexity of the transport by increasing the particle size. In Fig. 2(b) we show the filling of a magnetic lattice using large $(2.8 \mu \mathrm{m})$ particles, which reduces the size of the critical cluster to $N_{c}=0-4$ particles per unit cell. As a consequence, the filling process is much faster than before, and the colloidal front propagates by one composite cluster every second cycle rather than every fourth cycle. The net current produced by the large particles follows the same law $\tilde{j}=\tilde{\rho}-N_{c}$ as the small particles, but with $N_{c}=1$; i.e., exactly one particle per unit cell remains immobile while excess particles are transported.

In Fig. 2(d) we explore the dependence of $\tilde{j}$ on the amplitude of the normal component of the applied field $H_{z}$, for a fixed density $\tilde{\rho}=1.36$. No current is observed for amplitudes $H_{z}<H_{1}^{c}=780 \mathrm{~A} / \mathrm{m}$. Beyond the threshold $H_{1}^{c}$, only one particle per doublet is mobilized, while individual particles do not move, and the flux reaches the constant plateau $\tilde{j}=0.36$. Above a second threshold field, $H_{2}^{c}=1200 \mathrm{~A} / \mathrm{m}$, all particles are mobilized, and they flow at a constant speed above the lattice. The fields $H_{1}^{c}$ and $H_{2}^{c}$ are therefore the mobility edges where different subgroups of particles start to move. The plateaus in Fig. 2(d) correspond to $N_{c}=2,1,0$ fully occupied immobile sites. The rest of the particles are mobile and consist of fully and partially occupied sites contributing $\tilde{j}=\tilde{\rho}-N_{c}$ to the macroscopic current. There are six small interstitial regions around each magnetic bubble which can 
(a)

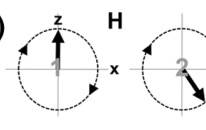

$\frac{\frac{2}{0}}{\frac{0}{0}}$

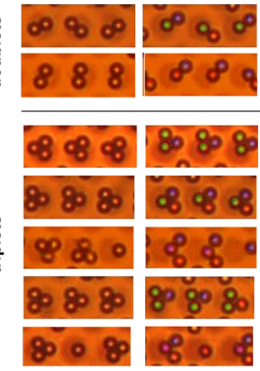

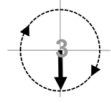
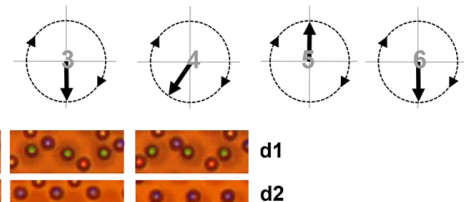

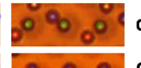

d1

d2

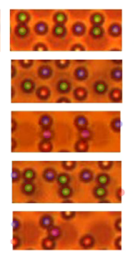

$8 \times 8 \times 4]^{t 1}$

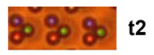

के क्ष $\mathrm{t}$
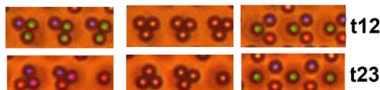

(b)

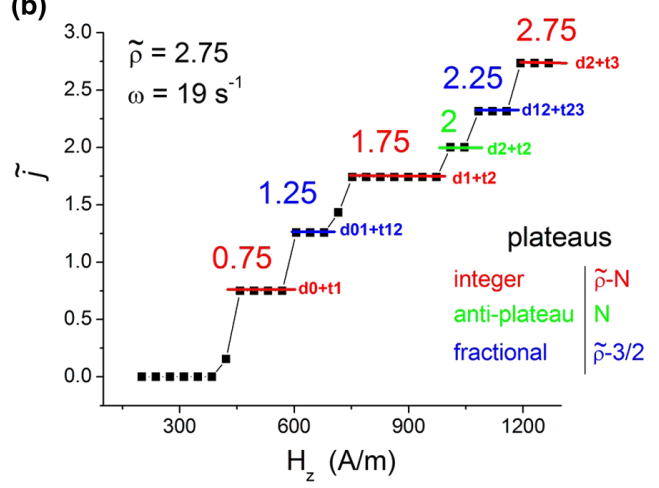

FIG. 3 (color online). (a) Polarization microscopy images showing the time evolution of the doublet $d$ modes and triplet $t$ modes. The orientation of the external magnetic field during the different stages is shown in the top row. In the images where interstitial wells exist, the stored particles are marked in green, particles in the type up or type down interstitial are marked in blue or red, respectively, and particles hopping from annihilated emitter bubbles are marked in purple. The $t 12$ and $t 23$ modes are period doubled modes, where the second cycle (columns 5 and 6) differs from the first cycle. (b) Dimensionless current $\tilde{j}$ as a function of $H_{z}$ for a density of $\tilde{\rho}=2.75$ exhibiting different kind of plateaus: partially filled integer plateaus in red, fully filled integer plateaus in green, and partially filled fractional plateaus in blue, together with the dominating modes (movie, ad Fig. 3.WMV [22]).

be separated into two types, Fig. 1(b) and 1(c). Type up and type down interstitials are marked in blue and in red, respectively, and are characterized by a neighboring bubble in the -21 and $2-1$ direction. In Figs. 2(a) and 2(b), there are equal numbers of particles flowing via the type up and type down interstitials, and the macroscopic current is unpolarized.

A rich variety of transport modes can be obtained by tuning the particle density and the magnetic field parameters. In Fig. 3(a) (movie, ad Fig. 3.WMV [22]) we show modes which arise for magnetic bubbles filled with doublets ( $d$ modes) and triplets ( $t$ modes). For field amplitudes $H_{z}<400 \mathrm{~A} / \mathrm{m}$, no particles are transported corresponding to the trivial $d 0$ and $t 0$ modes. The first modes giving rise to net particle current are the $d 1$ and $t 1$ mode. The $d 1$ mode occurs for fields in the range $700 \mathrm{~A} / \mathrm{m}<H_{z}<1100 \mathrm{~A} / \mathrm{m}$ and is characterized by the break up of a doublet into a stored particle in the bubble and an emitted particle in the interstitial. The emitted particle is transported via type up, marked in blue, or type down, marked in red, interstitials, and then collected by a magnetic bubble occupied by a single particle. During the next cycle, particles which were immobile during the first field cycle become mobile, and vice versa. The $t 1$ mode starts with a triplet of particles oriented with two corners toward the interstitials. Before the interstitial wells are nucleated, the triplets undergo a rotation by $\pi / 6$ followed by the emission of a single particle into one of the interstitial regions and the collection by a doublet with the final formation of a triplet having the initial orientation. In contrast, the $t 2$ mode starts with a particle triplet oriented with one corner between the interstitials followed by a rotation by $\pi / 6$ and an emission of two particles which simultaneously follow both types of interstitials towards the collector bubble. The $d 2$ and $t 3$ modes are fully mobile transport modes where all particles hop from the emitter to the collector bubble and are found for magnetic fields higher than $H_{z}>1200 \mathrm{~A} / \mathrm{m}$, that during the antiparallel orientation also annihilate the bubble wells. The triplet rotation takes more time than the actual particle hopping. Thus, increasing the driving frequency allows us to freeze the rotational motion, giving rise to two period doubling modes, $t 12$ and $t 23$. Since the hopping inverts the orientation of the triplets, two cycles are required to restore the original triplet orientation. For both modes, the number of particles hopping during the first and the second cycles are different, and this gives rise to an average fractional current of $3 / 2$ for the $t 12$ mode and $5 / 2$ for the $t 23$ mode. In Fig. 3(b) we show measurements of $\tilde{j}$ as a function of the field amplitude $H_{z}$ for a particle density $(\tilde{\rho}=2.75)$ for which the dominant colloidal clusters in the bubbles are doublets and triplets. An increase of $H_{z}$ leads to a superposition of doublet and triplet modes resulting in partially filled integer plateaus $(\tilde{\rho}-N)$, fully occupied integer plateaus $(N)$, and fractional partially filled plateaus $(\tilde{\rho}-3 / 2)$. Fractional plateaus occur due to period-doubled modes where first an odd and secondly an even number of particles are transported during the first and second field cycle. Further experiments produced tetramers and highly ordered clusters with even more complex transport modes and dynamics.

Our magnetic ratchet allows us to create polarized colloidal currents, using an additional field oriented along the -21 ( $y$ direction). We demonstrate this for the $d 1$ mode in Figs. 4(a) and 4(b), and for the $t 1$ mode in Figs. 4(c) and 4(d). When using the rotating field in the $(x, z)$ plane, both modes are characterized by a unpolarized current, with the emission of one particle which randomly hops towards the collector bubble via either the type up or type down interstitials. The application of an alternating field along 
(a)

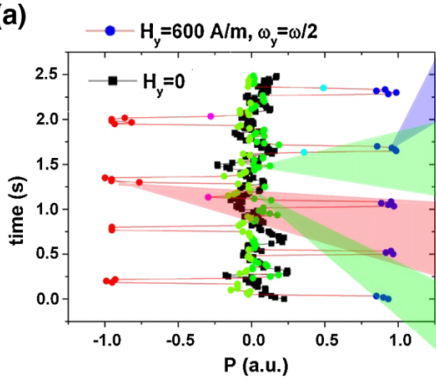

(c)

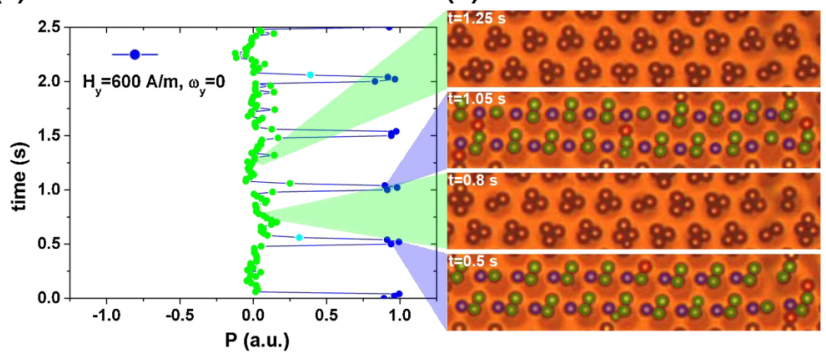

FIG. 4 (color online). (a) The polarization $P$ measured as a function of time without (squares) and with (circles) an additional field along the $y$ direction for the $d 1$ mode. (b) Four microscopy images corresponding to the first cycle doublet storage ( $t=1.25 \mathrm{~s})$, hopping in type down interstitial $(t=1.4 \mathrm{~s})$, second cycle doublet storage $(t=1.6 \mathrm{~s})$, and hopping in type up interstitial period ( $t=1.75 \mathrm{~s}$ ). (c) The polarization per unit cell measured as a function of time with the field along the $y$ direction for the $t 1$ mode. (d) Four microscopy images corresponding to the first cycle hopping $t=0.5 \mathrm{~s}$, triplet storage $t=0.8 \mathrm{~s}$, and a similar second cycle. Hopping particles are marked in blue or red when moving into an interstitial of type up or down, while particles stored in bubbles are marked in green (movie, ad Fig. 4.WMV [22]).

the $y$ direction, $H_{y}=H_{0} \sin \left(\omega_{y} t\right)$, phase locked to the rotating field with $\omega_{y}=\omega / 2=9.4 \mathrm{~s}^{-1}$, polarizes the particle emission in the $d 1$ mode, [Fig. 4(b), movie ad Fig. 4.WMV [22]]. With the $y$ field, the $d 1$ mode carries a macroscopic alternating polarized current, since the particles are periodically displaced in the type up and type down interstitials, Fig. 4(a). We created fully polarized direct currents by increasing the particle density and accessing odd triplet modes. We used a static field, $H_{y}=600 \mathrm{~A} / \mathrm{m}, \omega_{y}=0$ to displace the triplet such that the corner of the triplet before emission, lay close to the type up interstitial. The resulting macroscopic current of the $t 1$ mode displays an alternating polarization during each half cycle, Fig. 4(c). The alternating and direct polarization currents are collective effects only achieved with even and odd modes, respectively. The same principle is applied to the $t 12$ mode (not shown here): a $H_{y}$ field induces polarized hopping of single particles via type up interstitials during the first field cycle, while unpolarized hopping occurs during the second cycle when two particles are emitted to both the type up and down interstitials. This realizes a macroscopic fractional current since the net current flowing in the type up interstitial transports one particle per cycle while the current in the type down transports only half a particle per cycle.

In summary, our experiments show that excluded volume between mesoscopic particles gives rise to ratchet transport modes, where $n$ particle steps occur during a period consisting of $m$ cycles of the field, contributing with $\tilde{j}=$ $n / m$ to the particle current. For integer particle filling of the bubbles, only a single mode is selected. If the filling is incommensurate with the bubble lattice, a superposition of modes is observed due to the inhomogeneous distribution of composite clusters across the bubbles. The total current is the sum of the currents associated with each transport mode and remains at simple integer or fractional plateaus. The mobility or immobility of the partial particle layer determines whether one has a partially filled or fully filled plateau in the current.

We thank Tom H. Johansen for the FGF film and Matthias Schmidt for scientific discussions. P. T. acknowledges support from the ERC starting Grant "DynaMO" (No. 335040) and from the Programs No. RYC-201107605, and No. FIS2011-15948-E.

*ptierno@ub.edu

[1] S. E. Brown, G. Mozurkewich, and G. Grüner, Phys. Rev. Lett. 52, 2277 (1984)

[2] A. A. Middleton, O. Biham, P. B. Littlewood, and P. Sibani, Phys. Rev. Lett. 68, 1586 (1992).

[3] C. Reichhardt, F. Nori, Phys. Rev. Lett. 82, 414 (1999).

[4] A. B. Kolton, D. Domínguez, N. Grønbech-Jensen, Phys. Rev. Lett., 86, 4112 (2001).

[5] A. Vanossi, N. Manini, F. Caruso, G. E. Santoro, E. Tosatti, Phys. Rev. Lett. 99, 206101 (2007).

[6] T. A. Fulton, and G. J. Dolan, Phys. Rev. Lett. 59, 109 (1987).

[7] D. E. Grupp, T. Zhang, G. J. Dolan, N. S. Wingreen, Phys. Rev. Lett. 87, 186805 (2001).

[8] A. Yethiraj, A. van Blaaderen, Nature (London) 421, 513 (2003).

[9] D. Babic, C. Schmitt, C. Bechinger, Chaos 15, 026114(2005).

[10] H. Löwen, J. Phys. Condens. Matter 21, 474203 (2009).

[11] R. Bhat, S. N. Timasheff, Protein Sci. 1, 1133 (1992).

[12] P. G. Bolhuis, A. A. Louis, J. P. Hansen, Phys. Rev. Lett. 89, 128302 (2002).

[13] Y. Han, Y. Shokef, A. M. Alsayed, P. Yunker, T. C. Lubensky, and A. G. Yodh, Nature (London) 456, 898 (2008).

[14] D. T. N. Chen, Q. Wen, P. A. Janmey, J. C. Crocker, and A. G. Yodh, Annu. Rev. Condens. Matter Phys. 1, 301 (2010).

[15] J. Mattsson, H. M. Wyss, A. Fernandez-Nieves, K. Miyazaki, Z. Hu, D. R. Reichman, and D. A. Weitz, Nature (London) 462, 83 (2009).

[16] E. R. Weeks J. C. Crocker, A. C. Levitt, A. Schofield, and D. A. Weitz Science 287, 627 (2000).

[17] A. Stradner, H. Sedgwick, F. Cardinaux, W. C. K. Poon, S. U. Egelhaaf, and P. Schurtenberger, Nature (London) 432, 492 (2004).

[18] F. Jülicher, A. Ajdari, and J. Prost, Rev. Mod. Phys. 69, 1269 (1997). 
[19] P. Reimann, Phys. Rep. 361, 57 (2002).

[20] P. Hänggi, F. Marchesoni, Rev. Mod. Phys. 81, 387 (2009).

[21] P. Tierno, T. H. Johansen, T. M. Fischer, Phys. Rev. Lett. 99, 038303 (2007).
[22] See Supplemental Material at http://link.aps.org/ supplemental/10.1103/PhysRevLett.112.048302 for more experimental and theoretical details, and three video clips illustrating the particle dynamics. 